\title{
Epistemologische, literarische und kritische Funktionen des psychiatrischen Archivs
}

Michel Foucault hat mit dem Singular archive, der seit dem 16. Jahrhundert im Französischen kaum mehr verwendet wird, ein theoretisches Konstrukt bezeichnet, das von der Institution des Archivs unterschieden ist. Er definiert archive als „das allgemeine System der Formation und Transformation von Aussagen“ (Foucault 1992, 188). Diese Definition zielt weder auf ein empirisch gegebenes Korpus von Archivalien noch auf eine gedachte Totalität von Dokumenten, Spuren und Artefakten, noch gar auf die Aufbewahrungsstätte. „Ich werde“, so erläutert Foucault seinen Begriff,

als Archiv nicht die Totalität der Texte bezeichnen, die für eine Zivilisation aufbewahrt wurden, noch die Gesamtheit der Spuren, die man nach ihrem Untergang retten konnte, sondern das Spiel der Regeln, die in einer Kultur das Auftreten und Verschwinden von Aussagen, ihr kurzes Überdauern und ihre Auslöschung, ihre paradoxe Existenz als Ereignisse und Dinge bestimmen. (Foucault 2001, 902)

Wenn die Diskursanalyse das Gefüge der Regeln untersucht, mag sie zwar auf Archivbestände zurückgreifen, um zu rekonstruieren, wie ein Diskurs funktionierte, aber sie zielt nicht vorrangig auf die Analyse eines konkreten Archivs. Im psychiatrischen Diskurs spielen jedoch die Aufbewahrung und Speicherung von Dokumenten eine besondere Rolle. Aufbewahren und Speichern sind die Grundlage dessen, was im Folgenden als „Archivfunktion“ bezeichnet wird: Sie besteht darin, dass die Möglichkeit der Rekursion erzeugt wird. Man kann auf das Aufbewahrte wiederholt und immer wieder neu zugreifen.

Der Beitrag fragt, welche Rolle und Funktion der Möglichkeit einer Rekursion im psychiatrischen Diskurs zukommt. Das Archiv hat tief in die Erkenntnisbedingungen der Psychiatrie eingegriffen. Das Aufschreiben, Speichern und Prozessieren von Daten prägte maßgeblich die Arbeit in Klinik und Forschung. Die Techniken des Aufschreibens bildeten den Kern der psychiatrischen Methodenlehre, sie konstituierten psychiatrische Erkenntnisobjekte und strukturierten nicht zuletzt auch den Alltag in der Klinik. Parallel zur Verwissenschaftlichung der Medizin entwickelte die Psychiatrie aus den Aufschreibeverfahren der Ärzte im Zuge ihrer eigenen Verwissenschaftlichung und Ausdifferenzierung im Laufe des 19. Jahrhunderts ein eigenständiges Aufschreibesystem, das neben den Krankenakten 
auch Stations-, Medikations-, Labor-, Diagnosebücher umfasste und sich bis weit in die Infrastruktur der Klinik, die Publikationskultur der Disziplin, aber auch in die Justiz hinein verzweigte (vgl. Borck und Schäfer 2015). Dieses Aufschreibesystem stieg schließlich auch auf die Ebene des psychiatrischen Diskurses auf, dem es seinerseits Bedingungen und Regeln auferlegte. Die Möglichkeit einer Rekursion auf Akten schuf Voraussetzungen und Bedingungen des Forschens, die anderes und mehr waren als der Erfahrungsschatz eines Arztes, der über Jahre hinweg im Berufsleben aufgebaut wurde (vgl. Ledebuhr 2011). Um 1900 hatte die Archivfunktion schließlich in dem psychiatrischen Aufschreibesystem eine Schlüsselstellung inne, die sich jedoch in der Folge verselbständigte und zu Blockaden führte.

In seinen Analysen der psychiatrischen Aufschreibesysteme geht der vorliegende Beitrag nicht von einem einzelnen Archiv aus, das in einer psychiatrischen Institution angesiedelt wäre, sondern zeichnet in zwei Schritten nach, wie sich die Möglichkeit der Rekursion zunächst konfiguriert und dann in weiteren Schreibweisen fortsetzt und welche unterschiedlichen Funktionen sie jeweils herausbildet. In einem ersten Schritt wird die Neukonfiguration der Rekursion selbst am Beispiel von Emil Kraepelins doppeltem Archiv skizziert. Die Rekursion, so wird hier gezeigt, hat in die Voraussetzungen und Bedingungen eingegriffen, unter denen die Psychiatrie ihren Erkenntnisgegenstand konstituiert. Insofern steckt in der Archivfunktion auch eine epistemologische Funktion. Karl Jaspers' Kritik am Gebrauch des psychiatrischen Archivs richtet sich auf den Befund, dass die Schreibweisen, die mit der Rekursion einhergehen, diese epistemologische Funktion blockieren. In der Analyse dieser beiden psychiatriehistorischen Beispiele soll deutlich werden, dass die epistemologische Funktion selbst einem historischen Wandel unterliegt. In einem zweiten Schritt wird anhand von zwei literarischen Beispielen skizziert, wie die Archivfunktion in die Literatur eingeht und welche Dimensionen literarische Texte an ihr freisetzen. So soll schließlich angedeutet werden, wie die Literatur die Möglichkeit der Rekursion zu einer Kritik der Psychiatrie nutzt.

\section{2}

Im Zuge der Verwissenschaftlichung der Medizin in der zweiten Hälfte des 19. Jahrhunderts, die mit der Entdeckung der Ursachen insbesondere von Infektionen einherging, setzte sich die Auffassung durch, „daß Krankheiten spezifische Störungen seien, die bei allen Individuen gleichartige Veränderungen hervorrufen“ (Bleker 1979, 79). Auch wenn Krankheiten individuell variierten, schien ihr Verlauf hauptsächlich durch die sogenannte „Natur der Störung“ (Bleker 1979, 
79) bestimmt. Dieses kausale Modell wurde schließlich zum Inbegriff der Verwissenschaftlichung in der Medizin: Die Ursachen definierten Krankheiten und waren Grundlage für Aussagen über Verlauf und Ausgang der Erkrankungen.

Auch die Psychiatrie stützte sich auf dieses Modell. Man suchte nach den Ursachen der als Alienation, Irrsinn, Wahnsinn oder Geisteskrankheit bezeichneten Störungen im Gehirn und in den Nerven (Schott und Tölle 2006, 77). Der Psychiater Emil Kraepelin resümierte 1876 die Erwartungshaltung des Fachs wie folgt:

Wenn die einzige Ueberzeugung, welche heute wohl von allen Irrenärzten rückhaltlos geteilt wird, richtig ist, wenn wirklich alle Psychosen an krankhafte Prozesse in der Hirnsubstanz gebunden sind, dann dürfen wir den Nachweis pathologischer Spuren daselbst mit einer gewissen Wahrscheinlichkeit von einer besser gerüsteten näheren oder ferneren Zukunft erwarten. (Kraepelin 2003, 59-60)

Die psychiatrische Forschung selbst erfolgte entweder am Tiermodell: Man sezierte Tiere, um die Funktionsweise des Gehirns zu entschlüsseln. Oder man arbeitete auf der Grundlage eines post mortem erhobenen pathologischen Befunds, der an der Leiche aufzeigen sollte, welche Veränderungen im Gehirn des Erkrankten stattgefunden hatten.

Allerdings lieferte die Pathologie für zahlreiche Erkrankungen keine aussagekräftigen Ergebnisse. Die Erwartung, dass die Ursachen unmittelbar an den pathologischen Befunden zu demonstrieren wären, führte zu einer Blockade. Die Psychiatrie musste einen Umweg einschlagen. Kraepelin zog die Konsequenz hieraus, indem er ein empirisches Forschungsprogramm für die Psychiatrie formulierte, das sich auf drei Säulen stützte: auf die pathologische Anatomie, auf die Ergebnisse der experimentellen Psychologie und auf klinische Beobachtungen. Er trug die experimentelle Forschung in die Klinik hinein, holte die Patienten ins Labor und das Labor in die Klinik. Nicht zuletzt machte er so den Weg zum Einsatz technischer Medien zur Aufzeichnung in der Beobachtung der Patienten frei. Die Verbalisierung der Beobachtung mit bloßem Auge sollte durch die grafische Methode, durch Fotografie, Phonografie und schließlich Film ergänzt werden. Die technischen Medien nahmen indes nicht die Ordnung und Interpretation der Phänomene vorweg, sondern sie erzeugten mit ihrer Speicherfunktion zunächst einen Überschuss an Daten, denen durch die Archivoperation, d. h. eine Möglichkeit der Rekursion, zuallererst eine Struktur aufgeprägt werden musste, und entscheidend hierfür war die Krankenakte.

Seit dem 19. Jahrhundert hat die patientenbezogene Dokumentation Einzug in die Psychiatrie gehalten. Das Aufschreibeverfahren der Ärzte wurde aus der Registratur ausgegliedert, die hauptsächlich zu Verwaltungszwecken diente. Die klinische Akte war die „Schnittstelle zwischen medizinischem Wissen und indivi- 
dueller Erkrankung eines Menschen“ (Hess 2008, 44). Sie dokumentierte Anamnese und Krankheitsverlauf sowie die Therapie eines Patienten und besaß die offene Struktur eines Hefts, einer Blattsammlung oder eines Albums (vgl. Hess und Mendelsohn 1992). Zwar forderte die Verwaltung die Aufzeichnungen spezifischer Angaben, aber sie diktierte nicht mehr Art und Umfang des Eintrags. Vielmehr konnten zwischen den Deckeln einer Akte Schriftstücke und Objekte verschiedener Art versammelt werden. Eine Titelseite verzeichnete zumeist die Personalien, die Daten von Aufnahme und Entlassung, Transferierungen, die Diagnose, Angaben zum Kuratel usw. Die Akten selbst waren heterogene und unübersichtliche Dossiers: Sammlungsort von Äußerungen und Materialien jeglicher Art, die durch ihren „Bezug zum Patienten“ (Hess 2008, 45) vereinigt wurden.

Die Psychiatrie folgte in der Deutung dieser Dossiers den Spielregeln kasuistischer Wissensproduktion: Ein medizinischer Fall erlangte Aussagekraft nur mehr vor dem Hintergrund einer Gruppe von Fällen, mit denen er verglichen wurde. Auch wenn die Ärzte herausstellten, dass sie eine Kunst ausübten, die auf Erfahrungen beruhe, fasste die statistische Durchdringung des Wissens in Medizin und Psychiatrie Fuß. Allerdings konnten statistische Erhebungen allein keinen systematischen Überblick über die Fülle der Phänomene und Symptome gewähren, wie Kraepelin feststellte. Im Forschungsprozess selbst wurden die Beobachtungen und Daten häufig übersehen oder vergessen. Die Diagnose wiederum wirkte, wie Kraepelin weiterhin bemerkte, auf den klinischen Blick des Arztes zurück und vereitelte eine unvoreingenommene Interpretation der Beobachtungen. Schließlich war die Arbeit mit den Akten beschwerlich: Der Vergleich der Fälle erforderte unhandliche Synopsen, doch die Synopsen waren umso leistungsfähiger, je leichter die aufbereiteten Daten überschaubar waren. Der entscheidende Schritt, der es ihm erlaubte, die verwirrende Vielfalt zu ordnen, war die Errichtung eines Archivs, das mit einer neuen Art und Weise, die aufbewahrten Akten zu verarbeiten, gekoppelt wurde.

Das Archiv, das Kraepelin anlegte, sicherte zuallererst die Möglichkeit der Rekursion. Er ergänzte das bestehende psychiatrische Aufschreibesystem um sogenannte Zählkarten (vgl. Weber und Engstrom 1997). Die Zählkarte war ein standardisiertes Formular, auf dem für jeden aufgenommenen Patienten die „Personalien in knappster Form“ sowie „die wichtigsten Angaben über Ursachen und Entstehungsgeschichte, Erscheinungen, Verlauf und Ausgang seines Leidens“ (Kraepelin 1919, 239) verzeichnet wurden. Das Vorbild waren Formulare, die von den statistischen Ämtern des Reiches und der deutschen Länder für Erhebungen verwendet wurden. Kraepelin spielte, so darf man vermuten, immer wieder mögliche Gruppierungen der klinischen Einheiten und Untereinheiten mit den Karten durch: Es haben sich aus einem Bestand unbekannten Umfangs einige Hundert Karten erhalten, die aber nur einen kleinen Teil einer weitge- 
spannten Forschungsinfrastruktur ausmachen. Die Karten waren mobile Datenträger, die transkribiert und weiter verarbeitet werden konnten und so eine Überprüfung der Korrelation von Krankheitszeichen und Diagnosen erleichterten. Die Zählkarte fungierte als ein Datenfilter, der aus einer Krankengeschichte eine prägnante Struktur zurückbehält. Das Aufschreibesystem der Psychiatrie verfügte mit den Zählkarten über so etwas wie eine einfache, relationale Datenbank, die beliebige Vergleiche ermöglichte. So wirkte dieses doppelte System der möglichen Rekursion auf das eigentliche Archiv zurück. Kraepelin konnte nicht mehr nur punktuell Symptome und typische Stadien, sondern Verläufe und Verlaufsformen miteinander und wiederholt vergleichen.

Kraepelin hielt an der regulativen Idee fest, dass es eine Ursache und mithin gewisse Gesetzmäßigkeiten im Krankheitsgeschehen geben müsse. Das Archiv seiner experimentellen und klinischen Arbeiten gewährte einen synoptischen Überblick und ermöglichte eine statistische Durchdringung der Fälle. Es wurde zum unerlässlichen Hilfsmittel seiner Forschung und bildete die Voraussetzung für die Klassifikation von Erkrankungen durch deren Zusammenfassung nach Ähnlichkeiten in Symptomatik und Verlauf bzw. durch Aussonderung der Fälle, die sich einer Gruppenbildung entzogen. So konnte Kraepelin schließlich zwei Formenkreise von Erkrankungen gegeneinander und von allen anderen psychischen Störungen abgrenzen: das manisch-depressive Irresein und die Dementia praecox, die später als Schizophrenie bezeichnet wurde. Obwohl die Ätiologie der Krankheiten unbekannt war, schien die Einteilung nützlich und plausibel: „Die Heraushebung der manisch-depressiven und der Dementia-preacox-Gruppe und ihre gegenseitige Abgrenzung ist der bedeutsamste Fortschritt, den die systematische Psychiatrie je gemacht hat“ (Bleuler 1918, 286). Die Nosologie schloss eine Prognose des Krankheitsverlaufs ein und erlaubte somit die Kosten für die Heilung bzw. Pflege abzuschätzen; sie war Grundlage einer effizienten Auslastung von medizinischen Infrastrukturen, weil sie Kriterien für eine Verlegung von chronisch und unheilbar Erkrankten in Pflegeheime an die Hand gab; und sie diente nicht zuletzt als Handlungsanleitung für die ambulante und klinische Versorgung der Patienten.

\section{3}

Die Darstellung von psychiatrischen Fällen strukturierte das psychiatrische Wissen, insofern sie die Vermittlung des Einzelfalls mit den Diskursregeln leistete; sie generierte neue Fragestellungen, insofern sie Hypothesen anstieß; und sie brachte ein Wissen in Umlauf und setzte einen disziplinenübergreifenden Austausch in Gang. Die Krankheit selbst wurde wie eine Art oder Spezies aufge- 
fasst: Sie wurde dadurch diagnostiziert, dass an einer Gesamtheit von Inhalten nur das Gemeinsame festgehalten wurde. Die Merkmalsbildung stieg vom empirisch Gegebenen zu einer inhaltsärmeren Empirie auf. Diese Abstraktion vom Individuum war die Voraussetzung, dass das klinische Bild überhaupt als Einheit konstruiert und begrifflich bezeichnet werden konnte. Die psychiatrische Akte folgte der Ordnung des Personenstands: ein Fall, eine Akte. Die Fallgeschichten bzw. Krankengeschichten, die aus den Akten gewonnen wurden, waren um 1900 erzählende (nicht: literarische) Texte. Sie konnten knapp ausfallen, weil die Patienten die Repräsentanten einer Krankheit waren und sich am Einzelfall nichts Neues zeigte. Der psychiatrische Diskurs forderte keine ausführliche Darstellung.

Karl Jaspers (1883-1969) bemerkte, dass Diagnosen wie starre Bezeichnungsausdrücke funktionierten: Sie verrieten über den Gegenstand nichts, was man nicht schon wusste, wenn man die Gebrauchsregeln kannte, die der Bezeichnung zugrunde lagen. Der Name der Krankheit übte jedoch seine Erkenntnisfunktion desto besser aus, je mehr über das Bezeichnete bekannt war. Die klinischen Einheiten verleiteten zur Abkürzung der Diagnosestellung und blockierten die Forschung. Jaspers schlug deshalb vor, die klinisch-empirische Forschung auf ausführlichere Darstellungen zu verpflichten: Sie solle auf die „Gewinnung ganzer Lebensläufe“ abzielen, wie sie schon „Kraepelin immer gefordert“ (Jaspers 1910, 569) hatte. „Es liegt auf der Hand“, so rechtfertigte er seinen Vorschlag,

dass die Gewinnung guter Biographien nichts Alltägliches ist; in unzähligen Fällen bleiben wir auf allzu dürftige Angaben beschränkt. Es liegt ferner auf der Hand, dass, wenn einmal eine solche Biographie entsteht, sie die gewöhnliche Länge der Krankengeschichte übertreffen muß. [...] Für die Biographien in unserem Sinne pflegen wir ein nicht unerheblich größeres Material zu besitzen, als das, welches wir veröffentlichen. [...] Kurze Krankengeschichten erscheinen meist als ganz wertlos und überflüssig. (Jaspers 1910, 569)

Umfängliche Krankengeschichten würden hingegen, erstens, die Grundlage für beliebige Vergleiche schaffen und neue Forschungen anstoßen (Jaspers 1910, 568). Zweitens würden umfangreiche Krankengeschichten einen besseren Einblick in das Archiv der Psychiatrie gewähren:

Man kann sich in der Psychiatrie nicht verständigen ohne die Schilderung einzelner Fälle. Dies sind die Ecksteine, ohne die unsere Begriffsgebilde zusammenfallen. Das zeigt sich an der Wirkungslosigkeit so mancher älterer Arbeiten, die, weil die Fälle ja allgemein bekannt seien, auf diese oft pedantische und überflüssige, dazu arg raumfüllende Beigabe verzichten. (Jaspers 1910, 568)

Ausführliche Darstellungen von Lebensläufen und Biografien waren keine Neuheit. Nicht zuletzt in literarischen Texten und (Auto-)Biografien lag eine Sammlung von Lebensläufen vor, die in den sogenannten Psychopathografien 
auch ausgewertet wurden. Jedoch bestanden trotz ihres Reichtums an phänomenalen Beschreibungen Vorbehalte gegen literarische Texte. Sie galten als unbrauchbar für die klinische Forschung, denn sie standen unter dem Verdacht, bloße Fiktionen zu sein.

Die Darstellung der Krankengeschichte mittels seiner sogenannten ,biografischen Methode“ wollte jedoch anderes und mehr. Sie sollte eine Umschrift der Krankenakte leisten, ohne eine Vorselektion der Daten und Beobachtungen vorzunehmen. Die Erzählung des Psychiaters legte nur mehr eine transparente Folie über die Akte und gewährte dem Leser eine virtuelle Akteneinsicht. Der einzelne Patient samt seiner ganzen Biografie rückte darin ins Zentrum des Interesses: Die wissenschaftliche Objektivitätsforderung zielte nun nicht mehr nur auf das Typische einer Krankheit, sondern die Persönlichkeit des Kranken drückte insgesamt die Krankheit aus, die ihrerseits von hochgradiger Individualität war. Die epistemologische Schwelle, auf der die wissenschaftliche Erkenntnis begann, lag nicht mehr jenseits des Individuums, das ein Träger oder Repräsentant der Krankheit war, sondern verlief nunmehr mitten durch das Individuum.

Hatte Kraepelin die Rekursion genutzt, um eine Ordnung von Krankheitseinheiten $\mathrm{zu}$ etablieren, und damit die Archivfunktion $\mathrm{zu}$ einem Element eines datengestützten Vergleichssystems gemacht, so nutzte Jaspers die Rekursion, um eine Erkenntnisblockade aufzuheben: Es gab Fälle, die nicht fraglos in der Ordnung der Krankheitseinheiten ihren Platz fanden. Die nosologischen Einheiten hatten ihre Erkenntniskraft eingebüßt, sofern sie nur mehr einen Mechanismus zur begrifflichen Subsumption von Phänomenen am Laufen hielten. In der neuartigen Darstellungsweise des einzelnen Falls dagegen, die Jaspers vorschlug, zielte die Rekursion nicht vorrangig auf eine Extraktion von prägnanten Daten aus der Krankenakte, sondern auf eine Verbreiterung der Informationsbasis für die Synopse. Entscheidend war, dass auch dieser Modus der Rekursion auf die Akte nicht der Diagnose vorgriff. Man konnte die Biografie eines Patienten ausführlich erzählen, ohne bereits vorab zu wissen, welche Aspekte von Relevanz waren. Obgleich die biografische Methode sich wie die Zählkarte zwischen die Akte und die Diagnose einklinkte, diente sie weniger zur Herauslösung, Reduktion oder Purifizierung von Aufzeichungen, die als Daten weiterverarbeitet wurden, sondern zur Addition, Überlagerung und Verkomplizierung des Falls. Die Darstellung war nicht zuletzt an eine offene Zukunft bzw. künftige Psychiatrie adressiert. Das Erzählen senkte die epistemologische Schwelle ab, an der die Erkenntnis begann: Das Individuum war nicht als Vertreter oder Repräsentant einer bereits bekannten Krankheit, sondern als Träger einer noch unbekannten Krankheit von Interesse. Das erkrankte Individuum trat in das Feld des Wissens ein. 


\section{4}

Die Literatur verfügt über das Privileg, dass sie eine psychische Alterität fingieren kann. Doch stehen ihre Fiktionen dem tatsächlichen Erleben der Patienten und dem Alltag der Ärzte häufig fern. Wenn die literarischen Autorinnen und Autoren eine psychische Alterität hinstellen, greifen sie zumeist auf eigene Beobachtungen oder Erfahrungen zurück, oder aber sie nutzen Materialien, Dokumente, Zeugnisse und Archivalien. Jedoch besteht ein grundlegender Unterschied zwischen der literarischen und der wissenschaftlichen Indienstnahme der Archivfunktion. Auch wenn die epistemologische Funktion des psychiatrischen Archivs mit Eigennamen verknüpft wird, ist die Rekursion selbst eine anonyme Operation, die ein Glied in einer verteilten Handlungskette ist: Es spielt letztlich keine Rolle, wer die Rekursion ausführt. Die wissenschaftliche Zielsetzung legt fest, wie die Archivfunktion ausgeübt wird und welche Stellung sie in der Handlungskette einnimmt.

Seit den sechziger Jahren hat die Literatur Teil am Gegendiskurs der Antipsychiatrie, die eine berechtigte Kritik an den unhaltbaren Zuständen in den Anstalten und Kliniken übt. Im Folgenden sollen zwei Beispiele für einen literarischen Gegendiskurs kurz vorgestellt werden, die von Reinald Goetz und Heinar Kipphardt stammen. Ungeachtet der verschiedenen Stellungen, welche die Autoren in einer Geschichte der Psychiatriekritik innehaben, thematisieren beide Romane die psychiatrische Archivfunktion. Goetz erzählt in seinem Roman Irre (1983), wie der Psychiater Raspe in der Münchner Universitätsklinik seine berufliche Tätigkeit aufnimmt. ${ }^{1}$ „Doktor Raspe, der neue Kollege“ (Goetz 1983, 109), skizziert im Weitwinkel seiner ersten Beobachtungen im neuen Beruf eine emphatische Einheit von Forschung und Klinik. Er will über seine Erfahrungen in der Klinik schreiben und eine Kritik der Psychiatrie leisten. Er plant eine Kritik der Psychiatrie, die von deren Geschichte ausgeht: „Das hieße, die heutige Psychiatrie aus der gestrigen begreifen, nur so läßt sich die Zukunft einer humanen Psychiatrie entwerfen“ (Goetz 1983, 177). Auch wenn Raspes Plan keinen Bezug auf Michel Foucault nimmt, teilt seine Idee einer Analyse mit der Diskursanalyse, wie sie Foucault für die Psychiatrie in Angriff nimmt, die eigentliche Zielsetzung: Anhand der Geschichte der Psychiatrie wäre aufzuzeigen, welche Möglichkeiten verpasst, welche Chancen nicht ergriffen und welche Fehler begangen wurden, indem zum Beispiel nachgezeichnet würde, warum die Diagnostik von einem Instrument medizinischer Forschung zu einem bürokratischen Akt verkommen ist. Raspe wird rasch klar, dass die Aktenführung eine Prozedur ist, die ihren Zweck in der

1 Zum Verhältnis von Goetz’ Roman Irre zur Psychiatrie vgl. Rudolph (2008) und Hägele (2010). 
Administration, nicht aber in der Wissenschaft besitzt. Zwar waren in der formativen Phase der Psychiatrie zwischen 1870 und 1930 Diagnosestellung und Forschung noch eng verschränkt. Raspe bemerkt jedoch recht bald, dass 50 Jahre später die Diagnosestellung in der Klinik nur mehr als ein Abwehrmechanismus dient, der es nicht zuletzt ermöglicht, die alltägliche Arbeit zu bewältigen. Die Klinik kann mit solch einer Schwundform der Diagnostik deren Erkenntnisfunktion nicht ausschöpfen: „man müßte die Diagnostik historisch in ihr Recht setzen, man müßte sie wirklich wieder zu einem Instrument der Erkenntnis machen. Das wäre nicht unmöglich, schien es Raspe“ (Goetz 1983, 178). Raspe erfasst die epistemologische Funktion des Archivs: Die Forschung, die etwas Neues entdecken will, hat ihren Ausgangspunkt in einer Beschreibung der Phänomene, die ihren etablierten begrifflichen Rahmen zunächst einklammert. „Plötzlich schweigen in einem auch die Bücher. Beobachtung ist das Gegenteil einer geläufigen Terminologie“ (Goetz 1983, 116). Dem Projekt einer Wiedereinsetzung der Diagnostik zu einem Instrument der Forschung steht jedoch die Pragmatik alltäglicher Aktenführung entgegen. Die Diskrepanz zwischen einer Diagnostik, die „nur Selbstzweck war, um der Dokumentation etwa Genüge zu tun“ (Goetz 1983, 178), und einer Diagnostik, die dem Psychiater als „Ansporn“ (Goetz 1983, 177) dient, ist im Alltag der Klinik nicht zu überbrücken. Die Aktenführung bringt unter den Bedingungen des Klinikalltags einen Effekt hervor, der nicht intendiert ist.

Eine Krankengeschichte demonstriert ihre Wirklichkeitstreue mittels einer Darstellungskonvention. Nachträglich kann ihre Darstellung mit der Wirklichkeit verglichen, die referenzielle Bezugnahme überprüft und entschieden werden, ob sie wahrheitsgetreu ist oder nicht. Die Vorteile der Darstellungskonvention liegen auf der Hand: Die Darstellungen können zu einem späteren Zeitpunkt und von Dritten nachvollzogen werden. Die Verfahrensschritte der Aktenführung bringen im klinischen Alltag aber den Effekt hervor, dass die Darstellung von referenziellen Bezugnahmen entkoppelt werden kann. „Es geht nicht darum“, so erklärt ein erfahrener Kollege dem Anfänger Raspe,

„was du mit den Patienten wirklich, tatsächlich machst. Du kannst mit denen machen, was du willst, was du für richtig hältst. Aber in der Krankenakte, da mußt du alles konsistent erklären können, warum wozu. In der Krankenakte muß es revisionssicher sein, nur darum geht es, kontrollfest, falls es zu irgendwelchen Überprüfungen kommt, durch Oberarzt, Chef, oder gar juristischer Art. “ Nachdem Raspe dies verstanden hatte, war es keine Schwierigkeit mehr gewesen, kurze, konzis gefaßte Krankengeschichten zu diktieren, konzentriert auf Vollständigkeit und Plausibilität. (Goetz 1983, 214-215)

Denn knappe Ressourcen diktieren, wie die Akten geführt werden: „Die Krankengeschichten müssen kürzer werden, hieß es neulich in einer Konferenz. Das Schreibbüro sei überlastet“ (Goetz 1983, 81). Die Archivfunktion tritt in ihr reflexi- 
ves Stadium ein: Die Psychiater führen ihre Akten nicht mehr ausschließlich in der Absicht eigener Rekursion, sondern sie antizipieren die Möglichkeit einer Rekursion durch Dritte. In diesem reflexiven Stadium der Archivfunktion wird deren epistemologische Dimension versperrt. Das Aufschreibesystem der Psychiatrie bestimmt durch und durch, wie in der Psychiatrie zu schreiben ist, und lässt allenfalls Raum für „Usancen, die in ihrer Summe so etwas wie den Stil des Hauses prägen“ (Goetz 1983, 123). Der literarische Text von Goetz führt in die Ironie, dass die Archivfunktion ihre eigene epistemologische Dimension versperrt, weil sie sich gegen mögliche Kritik, die aus einer Rekursion durch Dritte hervorgehen könnte, $\mathrm{zu}$ immunisieren sucht. Innerhalb der psychiatrischen Institution scheint die Möglichkeit, mittels der Archivfunktion eine kritische Dimension zu eröffnen, blockiert. Das Archiv bietet zwar die Möglichkeit der Rekursion, aber die bloße Rückwendung auf Archivalien leistet noch keine Kritik.

\section{5}

Heinar Kipphardt hatte in seinem Fernsehspiel Leben des schizophrenen Dichters Alexander März (1975), im Roman März (1976) und im Theaterstück März. Ein Künstlerleben (UA 1980) die These einer strukturellen Verwandtschaft zwischen künstlerischer Schöpfung und Schizophrenie verfochten (vgl. Poore 1987, 198). Kipphardt, der selbst als Psychiater tätig war, errichtet im Roman März die Fiktion, dass er Materialien eines Archivs verwendet. Er verwendet den Kunstgriff der Archivfiktion, mit dem Goethe in den Wanderjahren, wie Hermann Broch formuliert, den „Grundstein der neuen Dichtung, des neuen Romans“ (Broch 1975, 87) legte, in kritischer Absicht. Es geht um das Leben des schizophrenen Dichters Alexander März, der Patient in der fiktiven Anstalt Lohberg ist. Der Roman montiert private Tagebucheinträge eines Psychiaters, Auszüge aus der Krankenakte des Patienten Alexander März, Berichte der Angehörigen und weitere Quellen. Kipphardts Roman geht „mit der literarischen Mode seiner Zeit“ (Wübben 2015, 3), insofern er in eine antipsychiatrische Kritik einstimmt, die mit voller Berechtigung ausspricht, dass die Zustände in den psychiatrischen Anstalten unhaltbar sind. Jedoch erfordert solch eine Kritik weder ein literarisches Schreiben, noch ist sie gar an die Archivfunktion geknüpft. Die kritische Funktion, die Kipphardts Romans ausübt, setzt an der Frage an, was überhaupt in das psychiatrische Archiv gelangt und wem welche Einsicht in die Archivalien gewährt wird. Die Institution blockiert die kritische Wendung der Archivfunktion. Kipphardt hingegen will herausfinden, auf welchen Erkenntnissen, Gewohnheiten und Denkweisen die herrschenden Praktiken in der Psychiatrie beruhen. Sein Roman 
weitet die Archivfunktion der Psychiatrie aus und verknüpft sie mit anderen Materialien, die gerade nicht im psychiatrischen Archiv aufbewahrt werden.

Der Roman setzt mit einem „Tagebucheintrag Koflers, Abteilungsarzt der psychiatrischen Landesklinik Lohberg“ (Kipphardt 2011, 7) ein. Die privaten Tagebucheintragungen des Arztes sind ihrerseits auf Materialien, die März hinterlassen hat, aber keinen Eingang in die Krankenakte gefunden haben, gestützt: „In der Kiste unter seinem Bett lag ein Zettel“ (Kipphardt 2011, 7). „Mein Schrifttum übereigne ich Dr. Kofler“ (Kipphardt 2011, 7), hat der abgängige Patient verfügt, der eine private Sammlung von Dokumenten angelegt hat. Der Roman präsentiert „Koflers Bericht“ (Kipphardt 2011, passim), zitiert aus der Krankenakte des Patienten März (Kipphardt 2011, 15, 17, 21 u. ö.). Er fingiert Ego-Dokumente (Kipphardt 2011, 19), Auszüge aus „Notizen“ (Kipphardt 2011, 20) des Arztes und Berichte von Angehörigen des Patienten (vgl. Kipphardt 2011, 51), Auszüge aus dem „Krankenbericht“ (Kipphardt 2011, 73) und Beobachtungen der Pfleger (Kipphardt 2011, 79, 85 u. ö.). Diese fingierte Rekursion auf Materialien und Archivalien ist mit Reflexionen auf deren Status und die rekursive Operation, die in der Psychiatrie mit den Akten ausgeführt wird, durchsetzt. Die Überlegungen betreffen die epistemologische Funktion der Krankenakten und Fallgeschichten: „Warnung. Alle Fallbeschreibungen sind in Richtung auf eine belegende Theorie gemacht, also mit falschem Bewußtsein“ (Kipphardt 2011, 111). Es geraten die pragmatischen Vorbehalte gegen Gewährung von Akteneinsicht in den Blick: „Beschluß, den Patienten Einblick in ihre Krankengeschichte zu geben und mit ihnen $\mathrm{zu}$ besprechen, wird von nahezu allen Pflegern kritisiert“ (Kipphardt 2011, 111). Nicht zuletzt wird das Bayerische „Verwahrungsgesetz“ (Kipphardt 2011, 68, 177) angeführt, das auch die Aufbewahrung und Weiterleitung von Ego-Dokumenten regelt.

Der Roman präsentiert also nicht nur Dokumente, die aus dem fiktiven Archiv der Klinik entstammen, sondern fingiert darüber hinaus zusätzliche Materialien, die keine andere Aufbewahrungsstätte besitzen als den Roman selbst. In der Archivfiktion scheinen dergestalt Fragen nach der psychiatrischen Archivfunktion auf: Welche Materialien werden auf welcher rechtlichen Grundlage aufbewahrt? Wer darf die Operation der Rekursion ausführen? Welche Effekte bringt die Rekursion für Ärzte, Patienten, Pfleger hervor? Das psychiatrische Archiv eröffnet die Möglichkeit einer Rekursion auf die Krankenakte, die ein Sammlungsort für Materialien jeglicher Art ist, die ihrerseits auf den Patienten bezogen sind. Kipphardt zielt darauf ab, dass die Spielregeln, die festlegen, was überhaupt in eine Akte gelangt, fragwürdig sind, indem er anderweitige Materialien präsentiert, die eben nicht in die Akte gelangen. Jaspers hatte zwar die Offenheit einer Akte für sämtliche Aspekte gefordert, die für den Fall von Relevanz sind. Die Archivfunktion wurde aber zumeist in vorhersehbarer Weise ausgeführt. Die Akte dokumentierte die Entwicklung der Krankheit und verzeichnet die Wirkungen 
der ärztlichen Behandlung. Hingegen nutzt Kipphardt die Archivfunktion, um die individuellen Züge des Patienten März herauszuarbeiten und seine Biografie in ein Ganzes von Umständen (Herkunft, Milieu, Stellung des Individuums in verschiedenen sozialen Gruppen) einzufügen. Während die Diskursart der Psychiatrie festlegt, was die Rekursion leisten soll, kann in der Literatur offenbleiben, worauf überhaupt zurückgegriffen wird. Man kann darin einen wesentlichen Unterschied zwischen dem Diskurs der Psychiatrie und der Literatur ausmachen: Die Psychiatrie folgt Regeln, die sowohl festlegen, was überhaupt in das Archiv gelangt, als auch festlegen, wie auf das Archiv zu rekurrieren ist. Die Literatur hingegen interessiert am Archiv, dass es die Möglichkeit einer regellosen Rekursion eröffnet. Kipphardt sucht mittels solch einer regellosen Rekursion die Kapselung des psychiatrischen Archivs aufzubrechen und hinterfragt die Selektionskriterien, die entscheiden, was überhaupt archiviert wird.

Kipphardt hat für seinen Roman nicht zuletzt auf Materialien zurückgegriffen, die ihm der Psychiater Leo Navratil zur Verfügung stellte. Navratil missbilligte Kipphardts „Verwendung der psychopathologischen Texte“ (Navratil 2011, 254), die der Autor „nicht bloß zu einer Anregung“ benutzt hat. Vielmehr hat Kipphardt, wie Navratil korrekt feststellt, „die verschiedensten Auszüge aus meinen [Navratils, Anm. A. S.] Büchern, auch Texte der verschiedensten Patienten, teils wortgetreu, teils entstellt, ohne Quellenangabe“ (Navratil 2011, 254) verwendet. Kipphardts Fiktion einer kritischen Archivfunktion ist nicht von moralischen, ethischen Fragen abzulösen, deren Diskussion eine eigene ausführliche Untersuchung erforderte (die der vorliegende Beitrag nicht leisten kann).

Jedenfalls erschöpft sich die psychiatrische Archivfunktion nicht in ihrer epistemologischen, kritischen und literarischen Dimension, die jeweils durch die Möglichkeit der Rekursion eröffnet werden. Weder können diese drei Aspekte der Archivfunktion streng unterschieden, noch kann ihre Unterscheidung unmittelbar an der Operation der Rekursion festgemacht werden. Die epistemologische Funktion kann sich der literarischen und kritischen Funktion annähern. Der Schluss, dass die drei Funktionen ohnehin konvergieren, wäre allerdings voreilig. Auch wenn die Diskursarten der Psychiatrie und der Literatur jeweils Rekursionen ausführen, bringt die gleiche Operation unterschiedliche Effekte hervor. Und keineswegs hat die Literatur die kritische Funktion gepachtet, sofern Kritik heißt, „herausfinden, auf welchen Erkenntnissen, Gewohnheiten und erworbenen, aber nicht reflektierten Denkweisen die akzeptierte Praxis beruht“ (Foucault 2005, 221). Ohnehin gehört das psychiatrische Archiv mittlerweile einer vergangenen Epoche an. Seit den achtziger Jahren des 20. Jahrhunderts wird das psychiatrische Archiv nach und nach in eine Datenbank transformiert, in der jede Rekursion in hohem Maße formalisiert ist und mit Operationen des Vergleichs und der Korrelation mit weiteren Daten verknüpft wird. An die Stelle der Archivfunktion 
ist die Erfassung, Dokumentation und statistische Durchdringung eines Datenbestands getreten.

\section{Literaturverzeichnis}

Bleker, Johanna. „Der Wandel der medizinischen Prognostik unter dem Einfluß der naturhistorischen Methode im 19. Jahrhundert“. Berichte zur Wissenschaftsgeschichte 2.1-2 (1979): 79-86.

Bleuler, Eugen. Lehrbuch der Psychiatrie. 2., erw. Aufl. Berlin: Julius Springer, 1918.

Borck, Cornelius, und Armin Schäfer (Hg.). Das psychiatrische Aufschreibesystem. Paderborn: Fink, 2015.

Broch, Hermann. „James Joyce und die Gegenwart“ [1936]. Kommentierte Werkausgabe. Bd. 9. Schriften zur Literatur 1: Kritik. Hg. Paul Michael Lützeler. Frankfurt a. M.: Suhrkamp, 1975. 63-91.

Engstrom, Eric J. Clinical Psychiatry in Imperial Germany. A History of Psychiatric Practice. Ithaca, London: Cornell University Press, 2003.

Foucault, Michel. Archäologie des Wissens. 5. Aufl. Frankfurt a. M.: Suhrkamp, 1992.

Foucault, Michel. „Über die Archäologie der Wissenschaft. Antwort auf den Cercle d’épistémologique“. Dits et Ecrits. Schriften I: 1954-1969. Hg. Daniel Defert und François Ewald unter Mitarbeit von Jacques Lagrange. Frankfurt a. M.: Suhrkamp 2001. 887-931.

Foucault, Michel. „Ist es also wichtig, zu denken?“. Dits et Ecrits. Schriften IV: 1980-1988. Hg. Daniel Defert und François Ewald unter Mitarbeit von Jacques Lagrange. Frankfurt a. M.: Suhrkamp, 2005. 219-223.

Goetz, Rainald. Irre. 2. Aufl. Frankfurt a. M.: Suhrkamp, 1983.

Hägele, Christoph. Politische Subjekt- und Machtbegriffe in den Werken von Rainald Goetz und Thomas Meinecke. Innsbruck, Wien, Bozen: StudienVerlag, 2010.

Hess, Volker. „Der Wandel der Krankengeschichte durch die Entwicklung der Krankenhausverwaltung. Ein altbekanntes Instrument im Wandel der Zeit“. Klinikarzt 37.1 (2008): 44-47.

Hess, Volker, und Andrew Mendelsohn. „Case and Series: Medical knowledge and paper technology, 1600-1900“. History of Science 48.3-4 (2010): 287-314.

Jaspers, Karl. „Eifersuchtswahn. Ein Beitrag zur Frage: ,Entwicklung einer Persönlichkeit‘ oder ,Prozeß‘?“. Zeitschrift für die gesamte Neurologie und Psychiatrie 1.1 (1910): 567-637.

Jaspers, Karl. Allgemeine Psychopathologie. Ein Leitfaden für Studierende, Ärzte und Psychologen. Berlin, Heidelberg: Julius Springer, 1913.

Kipphardt, Heinar. März. Roman und Materialien. 15. Aufl. Reinbek b. Hamburg: Rowohlt, 2011. Kraepelin, Emil. Psychiatrie. Ein Lehrbuch für Studierende und Ärzte. Bd. 1: Allgemeine Psychiatrie. 7., umgearb. Aufl. Leipzig: Johann Ambrosius Bart, 1903.

Kraepelin, Emil. Einführung in die psychiatrische Klinik. 3., völlig umgearb. Aufl. Leipzig: Johann Ambrosius Barth, 1916.

Kraepelin, Emil. „Die Erforschung psychischer Krankheitsformen“. Zeitschrift für die gesamte Neurologie und Psychiatrie 51.1 (1919): 224-246.

Kraepelin, Emil. „Die Richtungen der psychiatrischen Forschung. Vortrag, gehalten bei der Uebernahme des Lehramtes an der Kaiserlichen Universität Dorpat“ [1887]. Kraepelin in 
Dorpat 1886-1891. Hg. Wolfgang Burgmair, Eric J. Engstrom, Albrecht Hirschmüller und Matthias M. Weber. München: belleville, 2003. 55-80.

Ledebur, Sophie. „Schreiben und Beschreiben. Zur epistemischen Funktion von psychiatrischen Krankenakten, ihrer Archivierung und deren Übersetzung in Fallgeschichten“. Berichte zur Wissenschaftsgeschichte 34.2 (2011): 102-124.

Link, Jürgen. Versuch über den Normalismus. Wie Normalität produziert wird. Opladen: Westdeutscher Verlag, 1997.

Navratil, Leo. „Brief an Heinar Kipphardt, 15. April 1976“. Heinar Kipphardt. März. Roman und Materialien. 15. Aufl. Reinbek b. Hamburg: Rowohlt, 2011. 254-255.

Poore, Carol. „,Reportagen der Innenwelt‘: The Example of Heinar Kipphardt's März“. The German Quarterly 60.2 (1987): 193-204.

Roelcke, Volker. „Unterwegs zur Psychiatrie als Wissenschaft. Das Projekt einer ,Irrenstatistik“ und Emil Kraepelins Neuformulierung der psychiatrischen Klassifikation“. Psychiatrie im 19. Jahrhundert. Forschungen zur Geschichte von psychiatrischen Institutionen, Debatten und Praktiken im deutschen Sprachraum. Hg. Eric J. Engstrom und Volker Roelcke. Mainz: Schwabe, 2003. 169-188.

Risse, Guenter B., und John Harley Warner. „Reconstructing Clinical Activities: Patient Records in Medical History“. Social History of Medicine 5.2 (1992): 183-205.

Rudolph, Thorsten. irre/wirr: Goetz. Vom ästhetischen Terror zur systemischen Utopie. München: Wilhelm Fink, 2008.

Schott, Heinz, und Rainer Tölle. Geschichte der Psychiatrie. Krankheitsformen, Irrwege, Behandlungsformen. München: C. H. Beck, 2006.

Weber, Matthias, und Eric J. Engstrom. „Kraepelin's Diagnostic Cards. The Confluence of Empirical Research and Preconceived Categories“. History of Psychiatry 8.31 (1997): 375-385.

Wübben, Yvonne. Heinar Kipphardts Wahnsinnsstücke. Dokumentarisches und autobiographisches Schreiben. Berlin: Alpheus, 2015. 